Sociedad de Estadística e Investigación Operativa

Top (1998) Vol. 6, No. 2, pp. 313-319

\title{
Maximin location: Discretization not always works
}

\author{
Isabel Alonso, Emilio Carrizosa and Eduardo Conde \\ Departamento de Estadística e I.O. \\ Universidad de Sevilla \\ Tarfia s/n, 41012 Sevilla, Spain \\ isalon@cica.es, ecarriz@cica.es, educon@cica.es
}

\begin{abstract}
In this note we show by means of a simple example that, if the maximin problem with (nonlinear) concave increasing utility functions is solved by inspecting the extreme points of the (generalized) Voronoi diagram (as usually proposed), one may have to inspect an infinite number of candidate points.
\end{abstract}

Key Words: Maximin, Generalized Voronoi diagram, Discretization.

AMS subject classification: $90 \mathrm{~B} 85 ; 68 \mathrm{U} 05$.

\section{Introduction}

In this paper we present an example for which the discretization strategy frequently used for maximin location problems does not work judiciously.

The problem consists of finding a location on $S$ for a new obnoxious facility maximizing the minimum utility from the existing facilities, Dasarathy-White (1980), Erkut-Neuman (1989), Melachrinoudis-Cullinane (1985), Melachrinoudis-MacGregor (1995), Plastria (1996). In other words, we wish to solve the problem:

$$
\max _{x \in S} \min _{i=1, \ldots, n}\left\{q_{i}\left(\gamma_{i}(x)\right)\right\}
$$

where:

- The feasible set $S$ is a polygonal region in $\mathbb{R}^{2}$.

- Functions $\gamma_{i}, i=1, \ldots, n$ represent gauges, Rockafellar (1970), measuring the effects of the new facility on the demand points $v_{i}, i=$ $1, \ldots, n$, i.e.,

$$
\gamma_{i}(x)=\inf \left\{\lambda>0: \frac{1}{\lambda}\left(x-v_{i}\right) \in B_{i}\right\} \quad \forall x \in \mathbb{R}^{2} \quad \forall i=1, \ldots, n
$$

The research of the second and third authors is partially supported by Grant PB961416-C02-02 of Ministerio de Educación y Cultura, Spain

Received: December 1997; Accepted: October 1998 
where $B_{i}$ is a compact convex set with the origin of coordinates in its interior. The typical instance is the euclidean norm (each $B_{i}$ equals the unit circle, Dasarathy-White (1980), Melachrinoudis-Cullinane (1985), although nonsymmetric balls have also been suggested by Plastria (1996).

- Every demand point $v_{i}$ is associated with a non-decreasing concave utility function $q_{i}$ measuring the utility of the new service. The most studied case is the one with each $q_{i}$ being the identity, DasarathyWhite (1980), Okabe-Boots-Sugihara (1992), Okabe-Suzuki (1997), O'Rourke (1994), Preparata-Shamos (1985), although more realistic models can be obtained if utilities which are only concave are allowed, Erkut-Neuman (1989), Plastria (1996).

Since the objective function of $(P)$ does not enjoy good properties of convexity, a technique of global optimization is needed.

The most frequently used procedure, Dasarathy-White (1980), Melachrinoudis-Cullinane (1985), Melachrinoudis-MacGregor (1995), HakimiLabbé-Schmeichel (1992), consists of a discretization strategy which works by building explicit or implicitly the Voronoi diagram and evaluating its extreme points among which the candidate solutions to global optimum can be found.

The Voronoi region $\mathcal{V}\left(v_{i}\right)$ associated with $v_{i}$ is defined as follows:

$$
\mathcal{V}\left(v_{i}\right)=\left\{x \in S / q_{i}\left(\gamma_{i}(x)\right) \leq q_{j}\left(\gamma_{j}(x)\right), \forall j=1, \ldots, n,\right\}
$$

see e.g. Okabe-Boots-Sugihara (1992).

Based on this characterization, the following relation is satisfied at the Voronoi region $\mathcal{V}\left(v_{j}\right)$ :

$$
\min _{i=1, \ldots, n}\left\{q_{i}\left(\gamma_{i}(x)\right)\right\}=q_{j}\left(\gamma_{j}(x)\right) \quad \forall x \in \mathcal{V}\left(v_{j}\right)
$$

Hence, problem $(P)$ can also be expressed as:

$$
\max _{1 \leq j \leq n} \max _{x \in \mathcal{V}\left(v_{j}\right)} q_{j}\left(\gamma_{j}(x)\right)
$$


which, due to the increasing character of the utility functions $q_{j}$, reduces to:

$$
\max _{1 \leq j \leq n} q_{j}\left[\max _{x \in \mathcal{V}\left(v_{j}\right)} \gamma_{j}(x)\right]
$$

Hence, one must solve the $n$ optimization problems:

$$
\max _{x \in \mathcal{V}\left(v_{j}\right)} \gamma_{j}(x)
$$

Since each gauge considered $\gamma_{i}(x)$ is convex and the maximum of a convex

function on $\mathcal{V}\left(v_{i}\right)$ is attained at its extreme points, such points have to be examined to find an optimal solution.

This strategy has been successfully used for particular instances of problem $(P)$. Melachrinoudis and MacGregor (1995) address the problem for Euclidean distances, a planar polyhedral region consisting of $m$ faces and linear functions $q_{i}$, leading to an $O\left(m n^{2}\right)$ algorithm.

We present an example in which such method would lead to evaluate an infinite number of candidate points. We conclude, at this time, this method would be useless for some instances of problem $(P)$.

\section{An example}

We consider as feasible region the segment $S=\{(z, 0): z \in[0,1]\}$.

Let $\gamma_{1}=\gamma_{2}=\|\cdot\|$ be the Euclidean norm. Let $v_{1}=(0,1)$ and $v_{2}=$ $(0,-1)$.

The following constant $c=\frac{5}{4} \in[1, \sqrt{2}]$ and the concave increasing utility functions $q_{1}$ and $q_{2}$ are considered to satisfy the relation:

$$
\begin{gathered}
q_{1}\left(\left\|x-v_{1}\right\|\right)-q_{2}\left(\left\|x-v_{2}\right\|\right)= \\
=q_{1}(t)-q_{2}(t)=f(t)= \begin{cases}(t-c)^{5} \sin \left(\frac{1}{t-c}\right), & \text { if } t \in[1, \sqrt{2}] \backslash\{c\} \\
0, & \text { if } t=c\end{cases}
\end{gathered}
$$

Proposition 2.1. The previously defined function $f$ is twice continuously differentiable. 
It is needed the construction of the utility functions $q_{1}$ and $q_{2}$ that lead us to satisfy the concavity and increasing conditions as well as the relation $(R)$. The preceding assertion is needed in order to verify the conditions required for utilities functions.

Proposition 2.2. Let $\alpha$ and $\beta$ satisfy $\alpha>\sqrt{2} \beta \geq 0$. We consider:

$$
q_{2}(t)=-\frac{1}{2} \beta t^{2}+\alpha t
$$

The utility function $q_{2}$ is concave and increasing in $t \in[1, \sqrt{2}]$.

On the other hand, we study the conditions to be satisfied by the utility function $q_{1}$.

Proposition 2.3. Let $\alpha$ and $\beta$ be scalars such that:

$$
\alpha>\sqrt{2} \beta+\max _{t \in[1, \sqrt{2}]}\left|f^{\prime}(t)\right| \quad \text { and } \quad \beta \geq \max _{t \in[1, \sqrt{2}]}\left|f^{\prime \prime}(t)\right|
$$

if we consider:

$$
q_{1}(t)=f(t)+q_{2}(t)=f(t)-\frac{1}{2} \beta t^{2}+\alpha t
$$

then $q_{1}$ is concave and increasing, and also the relation $(R)$ is satisfied.

Indeed, since we have considered $\alpha$ satisfying

$$
\alpha>\sqrt{2} \beta+\max _{t \in[1, \sqrt{2}]}\left|f^{\prime}(t)\right|,
$$

then $f^{\prime}-\beta t+\alpha>f^{\prime}-\beta t+\sqrt{2} \beta+\max _{t \in[1, \sqrt{2}]}\left|f^{\prime}(t)\right| \geq 0$.

Hence $q_{1}^{\prime}(t)>0$, for all $t \in[1, \sqrt{2}]$, and the utility function $q_{1}$ is increasing.

Moreover $\beta \geq \max _{t \in[1, \sqrt{2}]}\left|f^{\prime \prime}(t)\right|$, thus $\beta \geq f^{\prime \prime}(t)$ for all $t \in[1, \sqrt{2}]$.

Hence, $q_{1}^{\prime \prime}(t) \leq 0$ for all $t \in[1, \sqrt{2}]$, and so we have shown its concavity. 
For instance, it is easily checked that

$$
\begin{aligned}
0.04 & >\max _{t \in[1, \sqrt{2}]}\left|f^{\prime}(t)\right| \\
\frac{3}{2} & >\max _{t \in[1, \sqrt{2}]}\left|f^{\prime \prime}(t)\right|,
\end{aligned}
$$

thus taking

$$
\begin{aligned}
\alpha & =3.1 \\
\beta & =2,
\end{aligned}
$$

problem $(P)$ has a single optimal solution due to the increasing character of utility functions $q_{1}$ and $q_{2}$ in $[1, \sqrt{2}]$. This solution is attained at $x=(1,0)$ while the discretization strategy of Voronoi diagrams leads us to an infinite number of function evaluations, as it is shown below.

In Figure 1 we have represented the Voronoi diagrams $\mathcal{V}\left(v_{1}\right)$ and $\mathcal{V}\left(v_{2}\right)$ for this instance.

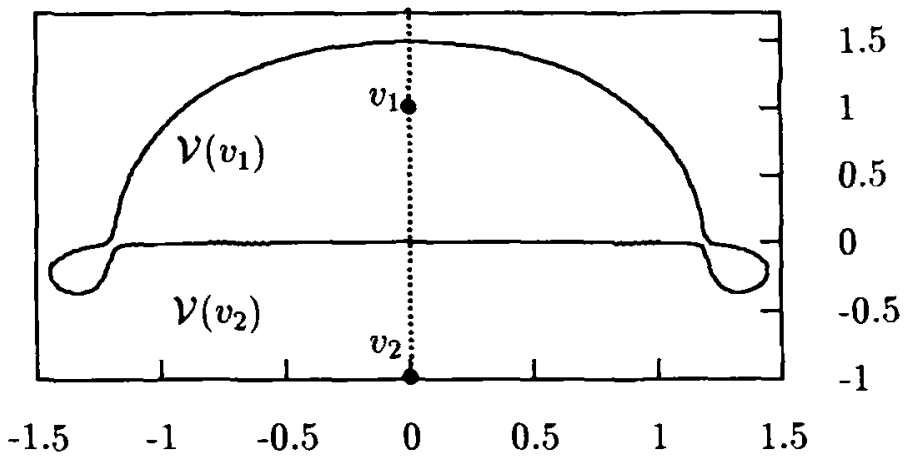

Figure 1: Voronoi diagram in the plane

The boundary of such Voronoi regions have a shape almost linear around the $Y$ axis. However, the true behaviour close to the $Y$ axis is sinusoidal, as clearly depicted in the detail of Figure 1 shown in Figure 2. In fact, the boundary of such Voronoi diagrams intersects the $Y$ axis (within the feasible region of our problem) in an infinity of points, thus the Voronoi diagram restricted to the feasible region $[0,1] \times\{0\}$ will have an infinite number of connected components. 


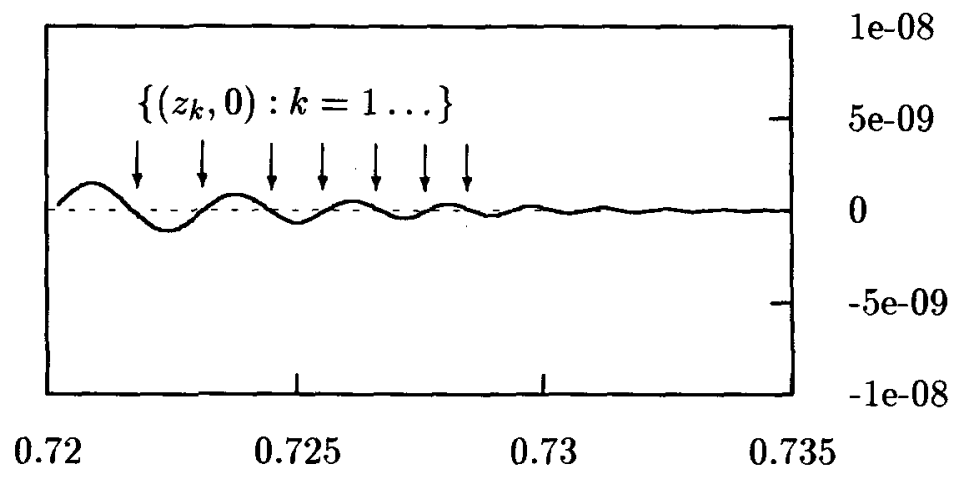

Figure 2: Voronoi diagrams. A detail

In fact, after some algebra, one obtains,

Proposition 2.4. For each $k \in \mathcal{Z}$ with $|k| \geq 2$ then, defining $z_{k}$ as

$$
z_{k}=\sqrt{\left(\frac{1}{k \pi}+c\right)^{2}-1} \in[0,1]
$$

it follows:

$$
\gamma_{1}\left(\left(z_{k}, 0\right)\right)=\gamma_{2}\left(\left(z_{k}, 0\right)\right)=\frac{1}{k \pi}+c
$$

so, from $(R)$ :

$$
q_{1}\left(\gamma_{1}\left(z_{k}, 0\right)\right)=q_{2}\left(\gamma_{2}\left(z_{k}, 0\right)\right)
$$

then $\mathcal{V}\left(v_{1}\right)$ and $\mathcal{V}\left(v_{2}\right)$ have infinitely many connected components, with the sequence $\left\{\left(z_{k}, 0\right)\right\}_{|k| \geq 2}$ as boundary points.

This yields the following: the equation $q_{1}\left(\gamma_{1}(x)\right)=q_{2}\left(\gamma_{2}(x)\right)$ has an infinite number of solutions, so an infinite number of function evaluations should be performed to obtain a global optimum among the candidate solutions.

In short, we have 
Proposition 2.5. Let $\alpha$ and $\beta$ be two scalars as follows:

$$
\alpha>\sqrt{2} \beta+\max _{t \in[1, \sqrt{2}]}\left|f^{\prime}(t)\right| \quad, \quad \beta \geq \max _{t \in[1, \sqrt{2}]}\left|f^{\prime \prime}(t)\right|
$$

hence, the utility functions $q_{1}$ and $q_{2}$ considered as above provide an example in which the frequently used discretization strategies to solve maximin problems are not effective, since in this case they lead us to an infinite number of function evaluations.

\section{References}

Dasarathy, B. and L.J. White (1980) . A maximum location problem. Operations Research, 28, 1385-1401.

Erkut, E. and S. Neuman (1989). Analytical models for Locating undesirable facilities. European Journal of Operational Research, 40, 275-291.

Hakimi, S.L. Labbé, M. and E. Schmeichel (1992). The Voronoi partition of a network and its implications in location theory. ORSA Journal on Computing, 4, $412-417$.

Melachrinoudis, E. and T.P. Cullinane (1985). Locating an undesirable facility within a geographic region using the MAXIMIN criterion, Journal of Regional Science, 25, 115-127.

Melachrinoudis, E. and J. MacGregor (1995). An $O\left(m n^{2}\right)$ algorithm for the Maximin problem in $E^{2}$. Operations Research Letters, 18, 25-30.

Okabe, A. Boots, B. and K. Sugihara (1992). Spatial Tessellations: Concepts and Applications of Voronoi Diagrams. Wiley, New York.

Okabe, A. and A. Suzuki (1997). Locational optimization problems solved through Voronoi diagrams. European Journal of Operational Research, 98, 445-456.

O'Rourke, J. (1994). Computational Geometry in C. Cambrigde University Press, Cambrigde.

Plastria, F. (1996). Optimal location of undesirable facilities: A selective overview. Belgian Journal of Operations Research, Statistics and Computer Science, 36, 109-127.

Preparata, F.P. and M.I. Shamos (1985). Computational Geometry. An introduction. Springer-Verlag, New York.

Rockafellar, R.T. (1970). Convex Analysis. Princeton University Press. 